\title{
Applications of Titanates in Electronics Technology
}

\author{
Renu Choithrani \\ Department of Physics and Electronics, Barkatullah University, Bhopal 462026, India
}

\begin{abstract}
Titanates have great attention mainly due to its versatile properties that make their various applications in science and technology such as in electronic transducers, thin film capacitors, computer memory cells, etc. The thermal study of ferroelectric titanates $\mathrm{RTiO}_{3}(\mathrm{R}=\mathrm{Pb}, \mathrm{La}, \mathrm{Ba})$ have been investigated for the first time using extended rigid ion model (ERIM) developed by the author.
\end{abstract}

Keywords-ferroelectric; titanates; thermal study; electronics technology

\section{INTRODUCTION}

Ferroelectric oxides with perovskite structure $\left(\mathrm{RTiO}_{3}\right)$, where $\mathrm{R}=\mathrm{Pb}, \mathrm{La}, \mathrm{Ba}$ show versatile properties [1] that make them promising for various applications in science and technology such as in electronic transducers, thin film capacitors, actuators, high-K dielectrics, pyroelectric sensors, nonlinear optics, computer memory cells, etc. Ferroelectric materials are a class of materials that possess high dielectric constants, relatively low dielectric loss, high electrical resistivity, moderate dielectric breakdown strength, and strong electromechanical and electrooptical behaviors $[2,3]$. One of the advantages in studying this particular class of titanate lies in its relatively simple electronic and lattice features; the materials undergo a succession of first order phase transitions from high to low temperatures characterized by a high symmetry cubic perovskite to slightly disordered ferroelectric structures with tetragonal, orthorhombic and rhombohedral symmetry. Orbital degrees of freedom, in addition to spin, charge, and lattice structure are gaining increasing interest in current solid-state physics. The studies on the thermal behavior of titanates are limited, since from theoretical point of view, a proper description of their thermal properties is still an area of active research. The thermal properties play an important role in the physics of materials as they characterize the behavior of the crystal in the field of external forces. We have developed and applied the extended rigid ion model (ERIM) [4] to compute the thermal properties such as cohesive, thermal and elastic properties of $\mathrm{PbTiO}_{3}, \mathrm{LaTiO}_{3}$, and $\mathrm{BaTiO}_{3}$ perovskite materials (with cubic $\mathrm{Pm} 3 \mathrm{~m}$ symmetry) as a function of temperature and discussed them in detail.

\section{COMPUTATIONAL DETAILS}

Extended Rigid Ion Model (ERIM) [4] has been developed by the author by incorporating the long-range (LR) Coulomb attraction, the short-range (SR) Hafemeister-Flygare (HF) type overlap repulsion effective up to the second neighbour ions, the van der Waals (vdW) attraction due to the dipoledipole $(\mathrm{d}-\mathrm{d})$ and dipole-quadrupole $(\mathrm{d}-\mathrm{q})$ interactions and zero point energy (ZPE) effects in the framework of Modified Rigid Ion Model (MRIM) developed earlier by us [5].
The framework of ERIM [4] is derived from the following interionic interaction potential:

$$
\emptyset_{\mathrm{ZRM}}=\emptyset_{\mathrm{MRNM}}+\emptyset_{\mathrm{zDZ}}
$$
by

Here, $\emptyset_{\text {MRLM }}$ potential (Renu Choithrani et al [5]) is given

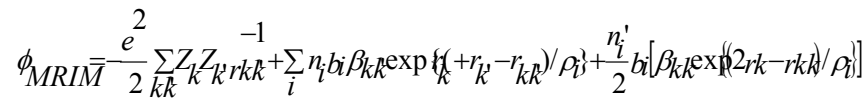

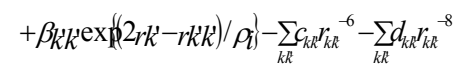

and

$$
\emptyset_{Z P E}=\left(\frac{9}{8}\right) N K_{D} \theta_{D}
$$

The symbols involved in equations (2) and (3) are the same as those defined in our earlier papers $[4,5]$. Here, $k\left(k^{\prime}\right)$ denote the positive (negative) ions and the sum is taken over all the ions $\left(k k^{\prime}\right)$.

In Eq. (2), the first term represents the long-range Coulomb attraction, the second and third terms are the shortrange Hafemeister-Flygare [6] type repulsion operating up to the second neighbour ions. The fourth and fifth terms in it are the vdW attraction energies due to the dipole-dipole $(d-d)$ and dipole-quadrupole $(d-q)$ interactions with $c_{k k}$, and $d_{k k}$, as the corresponding vdW coefficients.

The values of these coefficients are estimated by using the following expressions:

$$
\begin{gathered}
c_{k k^{\prime}}=\left(3 e^{2} \alpha_{k} \alpha_{k^{\prime}} / 2 m\right)\left[\left(\alpha_{k} / N_{k}\right)^{1 / 2}+\left(\alpha_{k^{\prime}} / N_{k^{\prime}}\right)^{1 / 2}\right]^{-1} \\
d_{k k^{\prime}}=\left(27 e^{2} \alpha_{k} \alpha_{k^{\prime}} / 8 m\right)\left[\left(\alpha_{k} / N_{k}\right)^{1 / 2}+\left(\alpha_{k} / N_{k^{\prime}}\right)^{1 / 2}\right]^{2} \\
{\left[\left(\alpha_{k} / N_{k}\right)^{1 / 2}+20 / 3\left(\alpha_{k} \alpha_{k}, / N_{k} N_{k^{\prime}}\right)\left(\alpha_{k^{\prime}} / N_{k^{\prime}}\right)\right]^{-1}}
\end{gathered}
$$

derived from the Slater-Kirkwood Variational (SKV) method [7]. Here, $m$ and $e$ are the mass and charge of electrons, respectively. $\alpha_{k}\left(\alpha_{k}\right)$ are the polarizabilities of $k\left(k^{\prime}\right)$ ions; $N_{k}\left(N_{k^{\prime}}\right)$ are the effective number of electrons responsible for the polarization of $k(k)$ ions. 
The values of $c_{k k^{\prime}}$ and $d_{k k^{\prime}}$ are evaluated using the Eqs. (4) and (5) and following the procedure prescribed by the authors $[4,5]$. Since the knowledge of interatomic interactions is of fundamental importance for describing the cohesive, thermal, transport, vibrational, elastic and numerous other physical properties of the solids. Thus, ERIM has been developed and applied by the author, probably for the first time, to describe the thermal properties of the perovskite materials. The main aim of ERIM is to reproduce the existing experimental data and to provide understanding of the interaction mechanism and the physical properties of materials. The computed results thus obtained are presented and systematically discussed below.

\section{RESUlTS AND DisCUSSION}

We have computed the model parameters $\left(\rho_{1}, b_{1}\right.$ and $\left.\rho_{2}, b_{2}\right)$ by taking the input data $[1-3,8-14]$ for $\mathrm{PbTiO}_{3}, \mathrm{LaTiO}_{3}$, and $\mathrm{BaTiO}_{3}$ and $\mathrm{vdW}$ coefficients $\left(\mathrm{c}_{\mathrm{kk}}\right.$, and $\mathrm{d}_{\mathrm{kk}}$ ) using the expressions [4] and [5] depicted in Table I. These model parameters are used to calculate the thermodynamical properties of $\mathrm{PbTiO}_{3} \mathrm{LaTiO}_{3}$, and $\mathrm{BaTiO}_{3}$ perovskites shown in Table II. It is found that the magnitude of cohesive energy for these perovskites are in closer agreement to the available measured values $[11,12]$. The negative values of the cohesive energy indicate the stability of the compound. The computed values of Reststrahlen frequency $\left(v_{0}\right)$ and Debye temperature $\left(\theta_{\mathrm{D}}\right)$ by ERIM shows consistency with the available data. [2, 8 , $11,12]$ (see Table II). The higher values of Debye temperature indicate the presence of higher phonon frequencies in $\mathrm{PbTiO}_{3}$, $\mathrm{LaTiO}_{3}$, and $\mathrm{BaTiO}_{3}$ titanate perovskites. The Grüneisen parameters $\gamma$ computed by ERIM lies in between 2 and 3 as observed by Dai et. al [13].

TABLE I. THE MODEL PARAMETERS OF PbTiO ${ }_{3}, \mathrm{LaTiO}_{3} \mathrm{AND} \mathrm{BaTiO}_{3}$

\begin{tabular}{|c|c|c|c|c|}
\hline \multirow[b]{2}{*}{$\mathbf{R}$} & \multicolumn{4}{|c|}{ Model Parameters } \\
\hline & $\begin{array}{l}\text { R-O } \\
\rho_{1}(\AA)\end{array}$ & $\begin{array}{c}\text { R-O } \\
b_{1}\left(10^{-12} \text { erg }\right)\end{array}$ & $\begin{array}{l}\text { Ti-O } \\
\rho_{2}(\AA)\end{array}$ & $\begin{array}{c}\text { Ti-O } \\
\mathrm{b}_{2}\left(10^{-12} \mathrm{erg}\right)\end{array}$ \\
\hline $\mathrm{Pb}$ & 0.6213 & 0.9989 & 0.8746 & 0.7123 \\
\hline $\mathrm{La}$ & 0.5798 & 0.9981 & 0.7475 & 0.6517 \\
\hline $\mathrm{Ba}$ & 0.4417 & 0.8328 & 0.6099 & 0.5078 \\
\hline
\end{tabular}

TABLE II. THERMAL PROPERTIES OF $\mathrm{PbTiO}_{3}, \mathrm{LaTiO}_{3}, \mathrm{AND} \mathrm{BaTiO}$

\begin{tabular}{cccc}
\hline \multicolumn{5}{c}{ Thermal Properties } \\
\hline $\begin{array}{c}\boldsymbol{\phi} \\
\mathbf{( e V / c e l l )}\end{array}$ & $\begin{array}{c}\mathbf{v}_{\mathbf{0}} \\
(\mathbf{T H z})\end{array}$ & $\begin{array}{c}\boldsymbol{\Theta}_{\mathbf{D}} \\
(\mathbf{K})\end{array}$ & $\boldsymbol{\gamma}$ \\
\hline-30.89 & 5.529 & 267.4 & 2.96 \\
$(-32.87)^{11}$ & $(5.174)^{11}$ & $(250.2)^{2}$ & $(2-3)^{13}$ \\
\hline-143.02 & 10.521 & 502.715 & 2.91 \\
$(-140.52)^{12}$ & $(10.764)^{12}$ & $(516.6)^{8}$ & $(2-3)^{13}$ \\
\hline-31.65 & 8.89 & 429.82 & 2.64 \\
$(-32.87)^{11}$ & $(8.87)^{11}$ & $(429.88)^{11}$ & $(2-3)^{13}$ \\
\hline
\end{tabular}

The specific heat as a function of temperature of $\mathrm{PbTiO}_{3}$, $\mathrm{LaTiO}_{3}$, and $\mathrm{BaTiO}_{3}$ have been shown graphically in Figures I, II and III using ERIM [4] and found that the specific heat curve follows the same trend of variation as that of available experimental specific heat values $[1,9,11]$.

It is interesting to note from Figures I, II and III that both the calculated and experimental specific heat values increase linearly with temperature indicating the display of phononic contributions $\left(\sim \mathrm{T}^{3}\right)$ in $\mathrm{PbTiO}_{3}, \mathrm{LaTiO}_{3}$ and $\mathrm{BaTiO}_{3}$ materials.

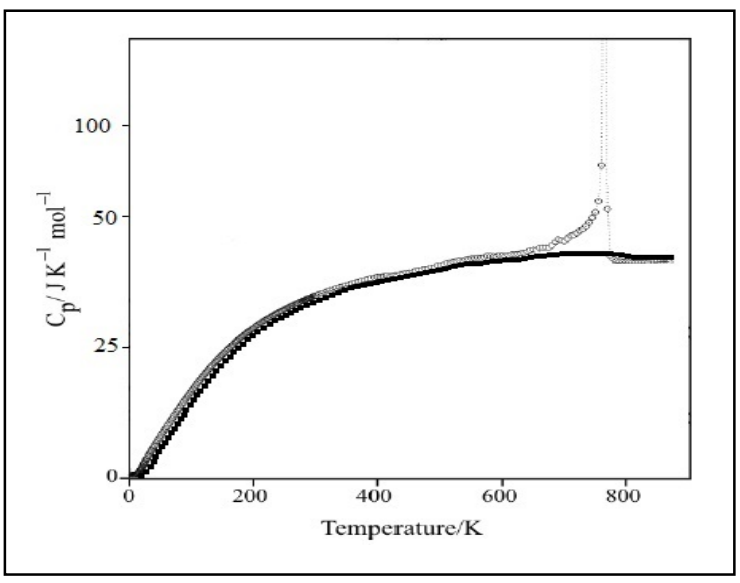

FIGURE I. VARIATION OF SPECIFIC HEAT WITH TEMPERATURE OF $\mathrm{PbTiO}_{3}$

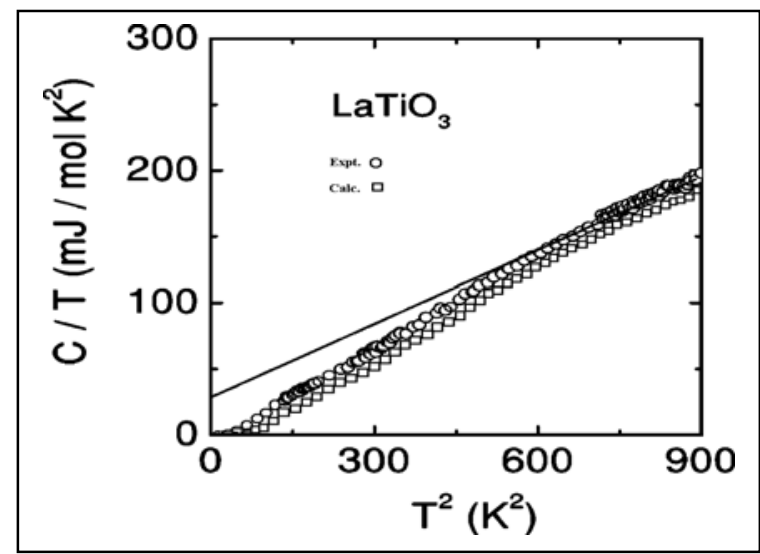

FIGURE II. VARIATION OF SPECIFIC HEAT WITH TEMPERATURE OF $\mathrm{LaTiO}_{3}$.

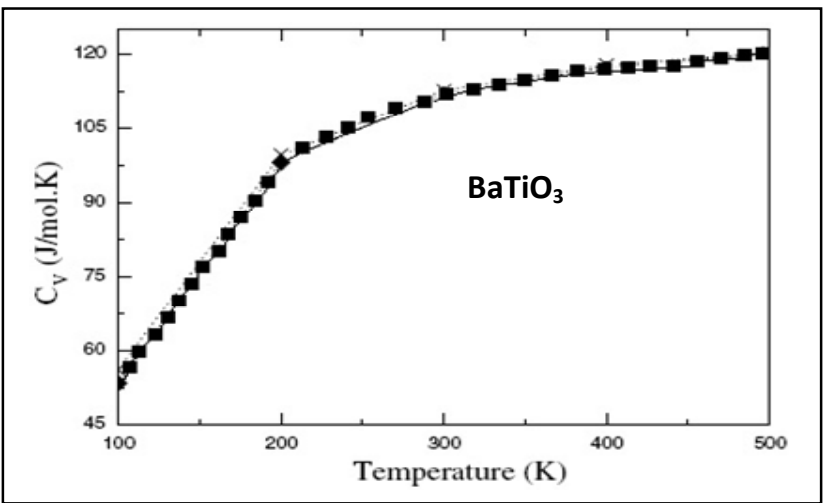

FIGURE III. VARIATION OF SPECIFIC HEAT WITH TEMPERATURE $\mathrm{OF} \mathrm{BaTiO}_{3}$

A sharp peak is observed at $764 \mathrm{~K}$ in experimental specific heat of $\mathrm{PbTiO}_{3}$ corresponding to the ferroelectric-paraelectric phase transition. This peak is not revealed from our theoretical results. This might be so because we have not included the 
magnetic contribution to the specific heat of $\mathrm{PbTiO}_{3}$. There is a sharp increase up to $\sim 300 \mathrm{~K}$ in $\mathrm{BaTiO}_{3}$ which is due to electronic contributions. Furthermore, the material displays roughly a similar behavior for the whole temperature range. The minor deviations toward lower temperatures can be further improved by additional magnons contribution to the specific heat of $\mathrm{PbTiO}_{3}, \mathrm{LaTiO}_{3}$, and $\mathrm{BaTiO}_{3}$.

\section{CONCLUSIONS}

It may be concluded that ERIM is effectively useful model and indeed one of the important tools for the unified and comprehensive study of the perovskite materials, because the beauty of our potential lies in satisfactory explanation of the variety of thermal properties with same success $[4,5]$ for the multiferroics, manganites and other perovskite materials. Hence, ERIM represents the realistic and qualitative applicability to describe the cohesive, thermal and elastic properties of the present titanate perovskites.

\section{ACKNOWLEDGMENT}

Renu Choithrani would like to acknowledge with thanks the Science and Engineering Research Board, Department of Science and Technology (DST), Government of India, New Delhi for providing the financial assistance and award under the Fast Track Young Scientist Scheme (SR/FTP/PS026/2010).

\section{REFERENCES}

[1] T. Yoshida, Y. Moriya, T. Tojo, H. Kawaji, T. Atake, and Y. Kuroiwa, "Heat capacity at constant pressure and thermodynamic properties of phase transitions in PbMO3 (M $=\mathrm{Ti}, \mathrm{Zr}$ and $\mathrm{Hf}) ", \mathrm{~J}$. Thermal Analysis and Calorimetry, vol. 95, pp. 675, 2009.

[2] M. Tachibana, and E. Takayama-Muromachi, "Thermal conductivity and heat capacity of the relaxor ferroelectric[PbMg1/3Nb2/3O3]1-x [PbTiO3]x", Phys. Rev. B, vol. 79, pp. 100104 (R) 2009.

[3] S. Piskunov, E. Heifets, R.I. Eglitis, and G. Borstel, " Bulk properties and electronic structure of $\mathrm{SrTiO} 3, \mathrm{BaTiO} 3, \mathrm{PbTiO} 3$ perovskites: an abinitio HF/DFT study", Comput. Mat. Sci., vol. 29, pp. 165, 2004.

[4] Renu Choithrani and N.K. Gaur, "Thermodynamics of Calcium Doped Perovskite Materials", AIP Conf. Proc. vol. 1447, pp. 1159, 2012; "Structural, Transport and Elastic properties of LaTiO3," AIP Conf. Proc., vol. 1512, pp. 1068, 2013; Transport studies of Nb- doped Pr0.7Sr0.3Mn1-xNbxO3 $(0 \leq \mathrm{x} \leq 0.05)$ manganites", Physica B: Condensed Matter, vol. 448, pp. 320, 2014.

[5] Renu Choithrani and N.K. Gaur, " Specific heat of Cd-doped manganites", J. Comput. Mat. Sci. vol. 49, pp. 107, 2010; Renu Choithrani, N.K. Gaur and R.K. Singh, " Study of calcium doping effect on thermophysical properties of some perovskite manganites", J. Alloys Compd. vol. 480: pp. 727, 2009.

[6] D. W. Hafemiester, and W.H. Flygare, " Outer-shell overlap integrals as a function of distance for halogen-halogen halogen-alkali and alkalialkali ions in the alkali halide lattice," J. Chem. Phys. vol. 43, pp. 795, 1965.

[7] J.C. Slater, and K.G. Kirkwood, " The van der Waals forces in gases," Phys. Rev. Lett. vol. 37, pp. 682, 1931.

[8] Chun-Mei Liu et al, "Structures, phase transition, elastic properties of $\mathrm{SnO} 2$ from first-principles analysis", Physica B, vol. pp.406: 1926, 2011

[9] V. Fritsch et al, " Magnetization and specific heat of LaTiO3", Phys. Rev. B, vol.65, pp. 212405, 2002.

[10] J. Hemberger et al, "Evidence for Jahn-Teller Distortions at the Antiferromagnetic Transition in LaTiO3" Phys. Rev. Lett. vol. 91, pp. $066403,2003$.
[11] N. Iles, A. Kellou, K. Driss Khodja, B. Amrani, F. Lemoigno, D. Bourbie, and H. Aourag, "Atomistic study of structural, elastic, electronic and thermal properties of perovskites $\mathrm{Ba}(\mathrm{Ti}, \mathrm{Zr}, \mathrm{Nb}) \mathrm{O} 3$, Comp. Mater. Sci. vol. 39, pp.896, 2007.

[12] Roger De Souza et al, " Formation and migration of cation defects in the perovskite oxide LaMnO3", J. Mater.Chem. vol. 9: pp.312, 1999.

[13] P. Dai, Zhang Jiandi, H. A. Mook, S.H. Lion, P.A. Dowben, and E.W. Plummer, " Experimental evidence for the dynamic Jahn-Teller effect in La0.65Ca0.35MnO3", Phys. Rev. B, vol.54, pp. R3694, 1996.

[14] Q.Z. Liu, H.F. Wang, F. Chen, and W. Wu, " Single-crystalline transparent and conductive oxide films with the perovskite structure: Sbdoped SrSnO3", J. Appl. Phys., vol. 103, pp.093709, 2008. 